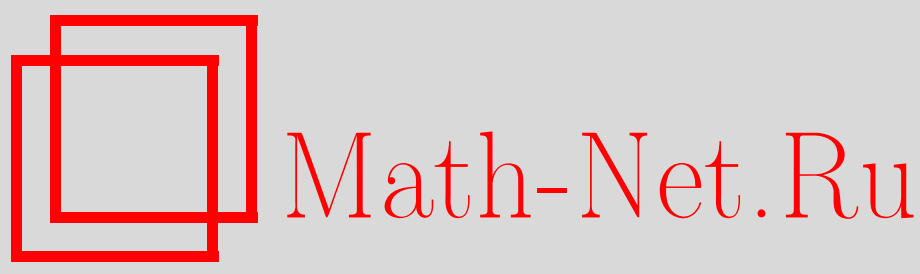

А. В. Аборнев, Нелинейные подстановки, рекурсивно порожденные над кольцом Галуа характеристики 4, Матем. вопр. криптогр., 2014, том 5, выпуск 4, 5-15

DOI: https://doi.org/10.4213/mvk132

Использование Общероссийского математического портала Math-Net.Ru подразумевает, что вы прочитали и согласны с пользовательским соглашением

http://www . mathnet.ru/rus/agreement

Параметры загрузки:

IP: 3.93 .64 .190

26 апреля 2023 г., 11:58:06

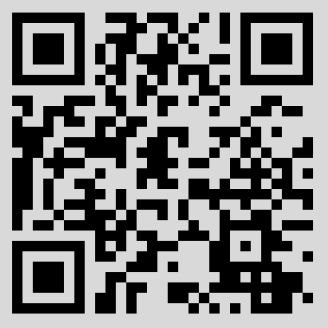


МАТЕМАТИЧЕСКИЕ ВОПРОСЫ КРИПТОГРАФИИ

2014 T. 5 № 4 C. 5-15

УДК 512.643

\title{
Нелинейные подстановки, рекурсивно порожденные над кольцом Галуа характеристики 4
}

\author{
А. В. Аборнев
}

ООО «Центр сертификационных исследований», Москва

Получено 22.IV.2014

Построен класс нелинейных подстановок $\pi_{F}$ на пространстве $\mathrm{GF}\left(2^{r}\right)^{m}$ произвольной размерности $m \geq 3$, рекурсивно-порожденных с законом рекурсии, определяемым многочленом $F(x)$ над кольцом Галуа $\operatorname{GR}\left(2^{2 r}, 4\right)$. Результаты работы А.А.Нечаева и автора [3] в данной работе обобщаются на случай произвольного кольца Галуа характеристики 4.

Ключевые слова: разрядно-подстановочный многочлен, кольцо Галуа.

Nonlinear permutations recursively generated over the Galois ring of characteristic 4

\section{A. V. Abornev}

LLC «Sertification Research Center», Moscow

Abstract. The class of nonlinear permutations $\pi_{F}$ of a space $\mathrm{GF}\left(2^{r}\right)^{m}$ of any dimension $m \geq 3$ is constructed. Each permutation $\pi_{F}$ is recursively generated by the characteristic polynomial $F(x)$ over the Galois ring $\operatorname{GR}\left(2^{2 r}, 4\right)$. Results of the paper by A.A. Nechaev and the author are generalized to an arbitrary Galois ring of characteristic 4.

Keywords: digit-permutable polynomial, Galois ring.

Citation: Mathematical Aspects of Cryptography, 2014, vol. 5, no. 4, pp. 5-15 (Russian)

(C) 2014 A. В. Аборнев 


\section{Введение}

Пусть $R=\operatorname{GR}\left(4^{r}, 4\right)$ - кольцо Галуа характеристики 4 и мощности $4^{r}$ с разрядным множеством $P=\Gamma(R)=\left\{\beta \in R \mid \beta^{q}=\beta\right\}, q=2^{r}$. Каждый элемент $a \in R$ имеет разложение

$$
a=a_{0}+2 a_{1}, \quad a_{s}=\gamma_{s}(a) \in P, \quad s=0,1 ;
$$

здесь $\gamma_{s}: R \rightarrow P(s=0,1)$ - разрядные функиии в разрядном множестве $P$. Алгебра $(P, \oplus, \cdot)$ с умножением, порожденным умножением в кольце $R$, и операцией сложения $a \oplus b=\gamma_{0}(a+b), a, b \in P$, является полем из $q=2^{r}$ элементов.

На векторном пространстве $P^{m}$ большой размерности $m$ строятся нелинейные подстановки с использованием семейства $L_{R}(F)$ линейных рекуррентных последовательностей с характеристическим многочленом

$$
F(x)=x^{m}-f_{m-1} x^{m-1}-\ldots-f_{1} x-f_{0} \in R[x] .
$$

Рассмотрим линейную рекуррентную последовательность (ЛРП) $u \in$ $L_{R}(F)$ с характеристическим многочленом $F(x) \in R[x]$. Ее знаки удовлетворяют равенству

$$
u(i+m)=\sum_{k=0}^{m-1} f_{k} u(i+k) .
$$

Каждый знак ЛРП $и$ имеет двоичное разложение

$$
u(i)=u_{0}(i)+2 u_{1}(i), \quad u_{0}(i), u_{1}(i) \in P, i=0,1, \ldots .
$$

Обозначим через $u[\overline{i, j}]$ отрезок $(u(i), u(i+1), \ldots, u(j))$ последовательности $u \in L_{R}(F)$ и через $L_{R}^{\prime}(F)$ множество всех таких линейных рекуррентных последовательностей $u \in L_{R}(F)$, что $u_{1}[\overline{0, m-1}]=\overline{0}$.

Рассмотрим отображение $\pi_{F}: P^{m} \rightarrow P^{m}$, заданное равенством

$$
\pi_{F}\left(x^{\downarrow}\right)=u_{1}[\overline{m, 2 m-1}]^{T},
$$

где $u_{1}$ строится по $u \in L_{R}^{\prime}(F), u_{0}[\overline{0, m-1}]^{T}=x^{\downarrow}$, и $T$ - оператор транспонирования.

Многочлен $F(x) \in R[x]$ степени $m$, для которого отображение $\pi_{F}$ является биекцией, будем называть разрядно-подстановочным (или $P П-$ многочленом). Будем говорить, что подстановка $\pi_{F}$ является рекурсивнопорожденной над кольцом $R$ с законом рекурсии $F(x)$. РП-многочлен назовем нетривиальным, если подстановка $\pi_{F}$ нелинейна. Таким образом, 
каждый РП-многочлен естественным образом задает семейство, вообще говоря, нелинейных подстановок на пространстве $P^{m}$. Подстановка $\pi_{F}$ на $\mathrm{GF}(q)^{m}$ естественным образом определяет подстановку $\tau_{F}$ на $\mathrm{GF}(2)^{r m}$.

Такой способ построения подстановок на пространстве предложен А.А. Нечаевым в [3], где приведен класс нетривиальных РП-многочленов над кольцом $\mathbb{Z}_{4}$. Еще один нетривиальный класс таких многочленов описан в [1]. Задача построения нелинейных подстановок на пространстве сколь угодно большой размерности, которые имеют компактное задание, рассматривалась также в [4].

Полученные результаты, как и в [3], применяются к криптографическому примитиву вида $\tau G$, где $G$ - регулярное представление группы, порожденной циклом $\left(0,1, \ldots, q^{m}-1\right)$, в $\mathfrak{S}\left(\mathrm{GF}(2)^{r m}\right)$. В качестве криптографических характеристик подстановки $\tau_{F}$ может рассматриваться порождаемая ею группа.

\section{1. Основные результаты}

Ниже описан класс нетривиальных РП-многочленов $F(x)$, индуцирующих нелинейные подстановки на алфавите $P^{m}$ сколь угодно большой мощности $2^{r m}$. Будем использовать обозначения

$$
F_{0}(x)=x^{m} \oplus \bigoplus_{i=0}^{m-1} \gamma_{0}\left(f_{i}\right) x^{i}, \quad F_{1}(x)=\bigoplus_{i=0}^{m-1} \gamma_{1}\left(f_{i}\right) x^{i}
$$

Теорема 1. Пусть $R=\mathrm{GR}\left(4^{r}, 4\right), F(x) \in R[x]-$ многочлен степени $m \geq 3$. Если $F_{0}(x)=x^{m} \oplus x^{m-1} \oplus x \oplus e$, то $F(x)$ является РПмногочленом тогда и только тогда, когда для каждого $\delta \in P$ верно равенство

$$
\left(\delta(x \oplus e) \oplus e \oplus F_{1}(x), F_{0}(x)\right)=e .
$$

Доказательство теоремы 1 приводится в п. 2.

Свойства смежного класса по циклической группе. Опишем свойства криптографического примитива, реализующего подстановки из множества $\tau G$, где $G$ - регулярное представление циклической группы $\left(\mathbb{Z}_{q^{m}},+\right)$, порожденной циклом $t=\left(0,1,2, \ldots, q^{m}-1\right)$, в $\mathfrak{S}\left(\mathrm{GF}(2)^{r m}\right)$. Отождествим вектор $v^{\downarrow}=\left(a_{1}, \ldots, a_{r m}\right)^{T} \in \mathrm{GF}(2)^{r m}, m>1$, с элементом $a=a_{1}+a_{2} 2+\ldots+a_{r m} 2^{r m-1} \in \mathbb{Z}_{2^{r m}}$. Пусть $F \in R[x]-$ РП-многочлен, и $\pi_{F}$ индуцирована многочленом $F(x)$. Тогда

$$
\pi_{F}\left(y^{\downarrow}\right)=\left(\psi_{1}\left(y^{\downarrow}\right), \ldots, \psi_{m}\left(y^{\downarrow}\right)\right)^{T}, \quad y^{\downarrow}=\left(y_{1}, \ldots, y_{m}\right)^{T} \in P^{m},
$$


где $\psi_{1}, \ldots, \psi_{m}$ - координатные функции. Если зафиксировать базис поля $\mathrm{GF}(q)$ как пространства над $\mathrm{GF}(2)$, то подстановке $\pi_{F}$ на $\mathrm{GF}(q)^{m}$ можно поставить в соответствие подстановку $\tau_{F}$ на $\mathrm{GF}(2)^{r m}$ с координатными функциями $\tau_{F}\left(x^{\downarrow}\right)=\left(\xi_{1}\left(x^{\downarrow}\right), \ldots, \xi_{r m}\left(x^{\downarrow}\right)\right)^{T}$,

Следующее утверждение следует из п. 1 теоремы 8 в [3].

Теорема 2. Пусть при введенных выше обозначениях первая координатная функиия $\xi_{1}$ подстановки $\tau_{F}$ существенно зависит от переменной $x_{r m}$. Если $r>1$, то $\left\langle\tau_{F} G\right\rangle=\mathfrak{S}\left(\mathrm{GF}(2)^{r m}\right)$.

Теперь рассмотрим свойства координатных функций подстановки $\pi_{F}$ из (7).

Предложение 3. РП-многочлен $F$ нетривиален тогда и только тогда, когда вектор $\left(\gamma_{0}\left(f_{0}\right), \ldots, \gamma_{0}\left(f_{m-1}\right)\right)$ содержит хотя бы два ненулевых элемента.

Доказательство. Из (3) следует, что для первой разрядной последовательности любой ЛРП $u \in L_{R}(F)$ выполнено равенство

$$
\begin{aligned}
u_{1}(i+m)=\bigoplus_{s=0}^{m-1} \gamma_{1}\left(f_{s}\right) u_{0}(i+s) \oplus \bigoplus_{s=0}^{m-1} \gamma_{0}\left(f_{s}\right) u_{1}(i+s) \oplus \\
\oplus \gamma_{1}\left(\sum_{s=0}^{m-1} \gamma_{0}\left(f_{s}\right) u_{0}(i+s)\right),
\end{aligned}
$$

где разрядная функция $\gamma_{1}\left(x_{1}+\ldots+x_{m}\right)$ имеет вид [2]

$$
\gamma_{1}\left(x_{1}+\ldots+x_{m}\right)=\sigma_{2}\left(x_{1}, \ldots, x_{m}\right)=\sum_{1 \leq s<t \leq m} x_{s} x_{t} .
$$

Следовательно, в (8)

$$
\gamma_{1}\left(\sum_{s=0}^{m-1} \gamma_{0}\left(f_{s}\right) u_{0}(i+s)\right)=\sigma_{2}\left(\gamma_{0}\left(f_{0}\right) u_{0}(i), \ldots, \gamma_{0}\left(f_{m-1}\right) u_{0}(i+m-1)\right)
$$

Опишем количество нелинейных координатных функций подстановки $\pi_{F}$. Обозначим через $T\left(F_{0}\right)$ период многочлена $F_{0}(x)$.

Предложение 4. Пусть $F(x) \in R[x]-$ разрядно-подстановочный многочлен. Тогда подстановка $\pi_{F}$ имеет ровно

$$
\min \left\{m, T\left(F_{0}\right)-m\right\}
$$

нелинейных координатных функиий. 
Доказательство. Пусть

$$
S(F)=\left(\begin{array}{ccccc}
0 & e & 0 & \ldots & 0 \\
0 & 0 & e & \ldots & 0 \\
& & & \ddots & \vdots \\
0 & 0 & 0 & \ldots & e \\
f_{0} & f_{1} & f_{2} & \ldots & f_{m-1}
\end{array}\right)
$$

- сопровождающая матрица многочлена $F(x) \in R[x]$. Из определения (5) следует, что действие подстановки $\pi_{F}$ можно описать также равенством

$$
\pi_{F}\left(x^{\downarrow}\right)=\gamma_{1}\left(S(F)^{m} x^{\downarrow}\right),
$$

где функция $\gamma_{1}$ применяется поэлементно.

Кроме того, если $A=S(F)^{m}=(a(i j)) \in R_{m, m}$ и $a(i j)=a_{0}(i j)+$ $2 a_{1}(i j) \in R, i, j \in \overline{1, m}$, то

$$
\psi_{k}\left(x^{\downarrow}\right)=\bigoplus_{s=0}^{m-1} a_{1}(k s) x_{s} \oplus \gamma_{1}\left(\sum_{s=0}^{m-1} a_{0}(k s) x_{s}\right), \quad k \in \overline{1, m} .
$$

Заметим, что функция $\psi_{k}\left(x^{\downarrow}\right)$ является нелинейной тогда и только тогда, когда в $k$-й строке матрицы $A_{0}=\gamma_{0}(A)$ есть хотя бы два ненулевых элемента. Если $m<T\left(F_{0}\right)<2 m$, то из равенства

$$
u_{0}[m, 2 m-1]^{T}=A_{0} \odot u_{0}[0, m-1]^{T},
$$

где $\odot-$ операция умножения матриц над полем $P$, получаем, что строки матрицы $A_{0}$ с номерами $T\left(F_{0}\right)-m+1, T\left(F_{0}\right)-m+2, \ldots, m$ являются строками единичной матрицы. Других строк веса 1 в матрице $A_{0}$ нет. Следовательно, линейными являются только координатные функции $\psi_{k}\left(x^{\downarrow}\right), k \in \overline{T\left(F_{0}\right)-m+1, m}$.

\section{2. Доказательство теоремы 1}

Нам понадобится следующий общий результат. Введем обозначения $S=\gamma_{0}(S(F)), \vec{F}_{j}=\gamma_{j}\left(\left(f_{0}, f_{1}, \ldots, f_{m-1}\right)\right) \in P^{m}, j=0,1$ и и

$$
g_{1}\left(x^{\downarrow}\right)=\sigma_{2}\left(\vec{F}_{0} \wedge x^{\downarrow}\right) \oplus \vec{F}_{1}^{\odot} x^{\downarrow},
$$

где $\wedge$ - операция покоординатного умножения векторов и $x^{\downarrow}=\vec{x}^{T}$. 
Лемма 5. Унитарный многочлен $F(x) \in R[x]$ степени $m$ является разрядно-подстановочным тогда и только тогда, когда система функuиü

$$
\left\{h_{i}\left(x^{\downarrow}\right)=g_{1}\left(x^{\downarrow} \odot\left(S^{T}\right)^{i-1}\right), \quad i=\overline{1, m},\right.
$$

над $P$ ортогональна.

Доказательство. Рассмотрим отображение $H=\left(h_{1}, \ldots, h_{m}\right): P^{m} \rightarrow$ $P^{m}$, определенное равенством (12), и отображение $\alpha: P^{m} \rightarrow P^{m}$, заданное по правилу

$$
\alpha\left(x_{0}, \ldots, x_{m-1}\right)=\left(y_{0}, \ldots, y_{m-1}\right)^{T}, \quad y_{i}=x_{i} \oplus \bigoplus_{k=0}^{i-1} f_{m-1-k, 0} x_{k} .
$$

Заметим, что $\alpha-$ биекция. Теперь для доказательства леммы достаточно показать, что

$$
\pi_{F}=\alpha^{-1} \circ H,
$$

где о - операция композиции отображений. Используем представление $\pi_{F}$ в виде (5). Пусть

$$
L_{R}^{\prime}(F)=\left\{u \in L_{R}(F): u_{1}[\overline{0, m-1}]=\overrightarrow{0}\right\} .
$$

Тогда в соответствии с (5) отображение $\pi_{F}$ полностью определяется условием

$$
\forall u^{\prime} \in L_{R}^{\prime}(F): \quad \pi_{F}\left(u_{0}^{\prime}[\overline{0, m-1}]^{T}\right)=u_{1}^{\prime}[\overline{m, 2 m-1}]^{T} .
$$

Из (3), используя разложение (1), получаем, что 1-я разрядная последовательность любой последовательности $u^{\prime} \in L_{R}(F)$ удовлетворяет соотношениям

$$
\begin{aligned}
u_{1}^{\prime}(i+m)=\left(\bigoplus_{k=0}^{m-1} f_{k 0} u_{1}^{\prime}(i+k)\right) \oplus & \\
& \oplus\left(\bigoplus_{k=0}^{m-1} f_{k 1} u_{0}^{\prime}(i+k)\right) \oplus \gamma_{1}\left(\sum_{k=0}^{m-1} f_{k 0} u_{0}^{\prime}(i+k)\right),
\end{aligned}
$$

и поэтому для последовательности

$$
v(i)=u_{1}^{\prime}(i+m) \oplus\left(\bigoplus_{k=0}^{m-1} f_{k 0} u_{1}^{\prime}(i+k)\right)
$$

выполнено равенство

$$
v(i)=\gamma_{1}\left(\sum_{k=0}^{m-1} f_{k 0} u_{0}^{\prime}(i+k)\right) \oplus \bigoplus_{k=0}^{m-1} f_{k 1} u_{0}^{\prime}(i+k) .
$$


Заметим, что из (17) и равенства $u_{1}^{\prime}[\overline{0, m-1}]=\overrightarrow{0}$ следует, что

$$
\begin{gathered}
v(i)=u_{1}^{\prime}(i+m) \oplus\left(\bigoplus_{k=m-i}^{m-1} f_{k 0} u_{1}^{\prime}(i+k)\right), \quad i \in \overline{0, m-1}, \\
v[\overline{0, m-1}]^{T}=\alpha\left(u_{1}^{\prime}[\overline{m, 2 m-1}]^{T}\right) .
\end{gathered}
$$

С другой стороны, используя (9) и (18), получаем, что

$$
v(i)=\sigma_{2}\left(\vec{F}_{0} \wedge u_{0}^{\prime}[\overline{i, i+m-1}]\right) \oplus \vec{F}_{1} \odot u_{0}^{\prime}[\overline{i, i+m-1}]^{T} .
$$

Из (3) следует, что

$$
u_{0}^{\prime}[\overline{i, i+m-1}]=u_{0}^{\prime}[\overline{0, m-1}] \odot\left(S^{T}\right)^{i}, i \geq 0 .
$$

Поэтому (20) дает

$$
v(i)=\sigma_{2}\left(\vec{F}_{0} \wedge\left(u_{0}^{\prime}[\overline{0, m-1}]^{\odot}\left(S^{T}\right)^{i}\right)\right) \oplus \vec{F}_{1} \odot S^{i} \odot u_{0}^{\prime}[\overline{0, m-1}]^{T} .
$$

Теперь, используя (11), имеем

$$
\begin{gathered}
v(i-1)=g_{1}\left(u_{0}^{\prime}[\overline{0, m-1}] \odot\left(S^{T}\right)^{i-1}\right)=h_{i}\left(u_{0}^{\prime}[\overline{0, m-1}]\right), \quad i=\overline{1, m}, \\
v[\overline{0, m-1}]^{T}=H\left(u_{0}^{\prime}[\overline{0, m-1}]^{T}\right) .
\end{gathered}
$$

Наконец, (14) следует из (19), (21), (15). Лемма 5 доказана.

Доказательство теоремы. Рассмотрим систему многочленов вида (12) для $F(x)$ и найдем условия, при которых она ортогональна. Ниже мы будем пользоваться тождествами $g_{1}\left(x^{\downarrow}\right)=g_{1}\left(x^{\downarrow}\right), \sigma_{2}\left(x^{\downarrow}\right)=\sigma_{2}\left(x^{\downarrow}\right)$. Пусть $F_{0}(x)=x^{m} \oplus x^{m-1} \oplus x \oplus e$. Тогда

$$
S=\left(\begin{array}{cccccc}
0 & e & 0 & \ldots & 0 & 0 \\
0 & 0 & e & \ldots & 0 & 0 \\
& \vdots & & \ddots & & \vdots \\
0 & 0 & 0 & \ldots & 0 & e \\
e & e & 0 & \ldots & 0 & e
\end{array}\right),
$$




$$
S^{i-1} \odot x^{\downarrow}=\left(\begin{array}{c}
x_{i} \\
x_{i+1} \\
\vdots \\
x_{1} \oplus x_{i} \oplus x_{m}
\end{array}\right), i \in \overline{2, m-1}, \quad S^{m-1} \odot x^{\downarrow}=\left(\begin{array}{c}
x_{m} \\
x_{1} \oplus x_{2} \oplus x_{m} \\
\vdots \\
x_{1} \oplus x_{m-1} \oplus x_{m}
\end{array}\right) \text {, }
$$

функция (11) имеет вид

$$
g_{1}\left(x^{\downarrow}\right)=\sigma_{2}\left(x_{1}, x_{2}, x_{m}\right) \oplus \vec{F}_{1} \odot x^{\downarrow}=\left(x_{1} \oplus x_{m}\right)\left(x_{1} \oplus x_{2}\right) \oplus x_{1} \oplus \vec{F}_{1} \odot x^{\downarrow} .
$$

Простыми вычислениями можно убедиться, что система (12) имеет вид

$$
\left\{\begin{array}{l}
h_{1}\left(x^{\downarrow}\right)=\left(x_{1} \oplus x_{m}\right)\left(x_{1} \oplus x_{2}\right) \oplus x_{1} \oplus \vec{F}_{1} \odot x^{\downarrow}, \\
h_{i}\left(x^{\downarrow}\right)=\left(x_{1} \oplus x_{m}\right)\left(x_{i} \oplus x_{i+1}\right) \oplus x_{i} \oplus \vec{F}_{1} \odot S^{i-1} \odot x^{\downarrow}, \quad i \in \overline{2, m-1}, \\
h_{m}\left(x^{\downarrow}\right)=\left(x_{1} \oplus x_{m}\right)\left(x_{1} \oplus x_{2}\right) \oplus x_{m} \oplus \vec{F}_{1} \odot S^{m-1} \odot x^{\downarrow} .
\end{array}\right.
$$

Рассмотрим отображение $H=\left(h_{1}, \ldots, h_{m}\right)^{T}: P^{m} \rightarrow P^{m}$ и введем обозначение

$$
S_{\vec{F}_{1}}=\left(\begin{array}{c}
\vec{F}_{1} \\
\vec{F}_{1} \odot S \\
\vdots \\
\vec{F}_{1} \odot S^{m-1}
\end{array}\right)
$$

Рассмотрим также разбиение пространства $P^{m}$ на непересекающиеся подмножества $\mathcal{A}_{\delta}=\left\{x^{\downarrow} \in P^{m}: x_{1} \oplus x_{m}=\delta\right\}, \delta \in P$. Тогда из (24) следует, что

$$
H\left(x^{\downarrow}\right)=\left(\delta(S \oplus E) \oplus E \oplus S_{\vec{F}_{1}}\right) \odot x^{\downarrow}, \quad x^{\downarrow} \in \mathcal{A}_{\delta} .
$$

Ввиду равенства $S_{\vec{F}_{1}}=F_{1}(S)$, полагая $v_{\delta}(x)=\left(\delta(x \oplus e) \oplus e \oplus F_{1}(x)\right)$, мы получаем, что

$$
H\left(x^{\downarrow}\right)=v_{\delta}(S) \odot x^{\downarrow}, \quad x^{\downarrow} \in \mathcal{A}_{\delta} .
$$

В соответствии с леммой 5 нам необходимо доказать, что $H-$ биекция тогда и только тогда, когда выполнено условие (6).

Перейдём к непосредственному доказательству теоремы. Положим $e^{\downarrow}=(e, \ldots, e)^{T}$. Опишем элементы множеств $A_{\delta}, \delta \in P$.

Лемма 6. Для каљдых $x^{\downarrow} \in P^{m} u \delta \in P$ следующие условия равносильubl:

(a) $x^{\downarrow} \in \mathcal{A}_{\delta}$; 
(б) $\left(S^{m-1} \oplus E\right) \odot x^{\downarrow}=\delta e^{\downarrow}$;

(в) $x^{\downarrow}=(S \oplus E) \odot y^{\downarrow} \oplus(\delta, 0, \ldots, 0)^{T}$ для некоторого $y^{\downarrow} \in P^{m}$.

Доказательство. (а) $\Rightarrow$ (б) Достаточно заметить, что первая строка матрицы $\left(S^{m-1} \oplus E\right)$ есть $(e, 0, \ldots, 0, e)$.

$(б) \Leftrightarrow($ в) Каждый вектор вида (в) удовлетворяет условию (б), потому что $\left(S^{m-1} \oplus E\right) \odot(S \oplus E)=F_{0}(S)=0$. Так как $\operatorname{rank}(S \oplus E)=m-1$ и $\left(S^{m-1} \oplus E\right) \neq 0$, то количество векторов, удовлетворяющих условию (в), равно количеству решений уравнения (б).

(б) $\Rightarrow$ (а) Очевидно, так как в (б) $\delta e=x_{1} \oplus x_{m}$.

Лемма 7. Отображение $H$ биективно тогда и только тогда, когда $F_{1}(e) \neq e$ и для каждого $\delta \in P$ выполнено равенство

$$
H\left(A_{\delta}\right)=A_{\delta\left(e \oplus F_{1}(e)\right)} \cdot
$$

Доказательство. Очевидно, что если $F_{1}(e) \neq e$ и выполнены равенства в условии леммы, то $H$ биективно.

Докажем в обратную сторону. Рассмотрим действие отображения $H$ на множествах $\mathcal{A}_{\delta}, \delta \in P$. Пусть $x^{\downarrow} \in \mathcal{A}_{\delta}$. По пункту (в) леммы 6

$$
\begin{aligned}
& H\left(x^{\downarrow}\right)=v_{\delta}(S) \odot\left((S \oplus E) \odot y^{\downarrow} \oplus(\delta, 0, \ldots, 0)^{T}\right)= \\
& v_{\delta}(S) \odot(S \oplus E) \odot y^{\downarrow} \oplus v_{\delta}(S) \odot(\delta, 0, \ldots, 0)^{T}= \\
& \quad(S \oplus E) \odot\left(v_{\delta}(S) \odot y^{\downarrow}\right) \oplus v_{\delta}(S) \odot(\delta, 0, \ldots, 0)^{T} .
\end{aligned}
$$

Пользуясь определением многочлена $v_{\delta}(x)$, преобразуем последнее слагаемое:

$$
\begin{aligned}
& v_{\delta}(S) \odot(\delta, 0, \ldots, 0)^{T}=\left(\delta(S \oplus E) \oplus E \oplus F_{1}(S)\right) \odot(\delta, 0, \ldots, 0)^{T}= \\
& \delta(S \oplus E) \odot(\delta, 0, \ldots, 0)^{T} \oplus\left(E \oplus F_{1}(S)\right) \odot(\delta, 0, \ldots, 0)^{T} .
\end{aligned}
$$

Подставляя последнее равенство в (29), получаем, что

$$
H\left(x^{\downarrow}\right)=(S \oplus E) \odot\left(v_{\delta}(S) \odot y^{\downarrow} \oplus \delta(\delta, 0, \ldots, 0)^{T}\right) \oplus\left(E \oplus F_{1}(S)\right) \odot(\delta, 0, \ldots, 0)^{T} .
$$

Умножим обе части полученного равенства на матрицу $\left(S^{m-1} \oplus E\right)$ и воспользуемся тождеством $\left(S^{m-1} \oplus E\right) \odot(S \oplus E)=0_{m \times m}$. Тогда

$$
\begin{aligned}
\left(S^{m-1} \oplus E\right) \odot H\left(x^{\downarrow}\right)= & \left(S^{m-1} \oplus E\right) \odot\left(E \oplus F_{1}(S)\right) \odot(\delta, 0, \ldots, 0)^{T}= \\
& \left(E \oplus F_{1}(S)\right) \odot\left(S^{m-1} \oplus E\right) \odot(\delta, 0, \ldots, 0)^{T}=\left(E \oplus F_{1}(S)\right) \odot e^{\downarrow} \delta .
\end{aligned}
$$


Преобразуем последнее равенство. По определению матрицы $F_{1}(S)=$ $S_{\vec{F}_{1}}$, и так как $\vec{F}_{1} \odot e^{\downarrow}=F_{1}(e)$ и $S \odot e^{\downarrow}=e^{\downarrow}$, то

$$
F_{1}(S) \odot e^{\downarrow}=\left(\begin{array}{c}
\vec{F}_{1} \odot e^{\downarrow} \\
\vec{F}_{1} \odot S \odot e^{\downarrow} \\
\vdots \\
\vec{F}_{1} \odot S^{m-1} \odot e^{\downarrow}
\end{array}\right)=F_{1}(e) e^{\downarrow} .
$$

Отсюда $\left(S^{m-1} \oplus E\right) \odot H\left(x^{\downarrow}\right)=\delta\left(e \oplus F_{1}(e)\right) e^{\downarrow}$. По пункту (б) леммы 6 при любом $F_{1}(x)$

$$
H\left(\mathcal{A}_{\delta}\right) \subset \mathcal{A}_{\delta\left(e \oplus F_{1}(e)\right)}, \quad \delta \in P .
$$

Если $H$ биективно, то отсюда получаем требуемое условие.

Заметим, что условие (6) означает, что все матрицы $v_{\delta}(S), \delta \in P$, обратимы. Теперь, пользуясь доказанной выше леммой, получаем, что если выполнено условие теоремы (6), то $H$ - подстановка. Действительно, при этом выполнено условие $F_{1}(e) \neq e$, так как $\left(F_{1}(x) \oplus e, F_{0}(x)\right)=e$.

Обратно, пусть $H$ - биекция. Покажем, что (6) верно. По доказанному выше $H\left(\mathcal{A}_{\delta}\right)=\mathcal{A}_{\delta\left(e \oplus F_{1}(e)\right)}, \quad \delta \in P$. Очевидно, что тогда $F_{1}(e) \neq e$. Предположим, что $\left(v_{0}(x), F_{0}(x)\right) \neq e$. Тогда $v_{0}(S)$ не обратима, но из $(27)$ имеем $\operatorname{Ker} v_{0}(S) \cap \mathcal{A}_{0}=\left\{0^{\downarrow}\right\}$. Поэтому для некоторого $\delta \in P$ существует такой $\eta^{\downarrow} \in \mathcal{A}_{\delta}$, что $v_{0}(S) \odot \eta^{\downarrow}=0^{\downarrow}$, и из соотношения $v_{\delta}(S)=\delta(E \oplus S) \oplus v_{0}(S)$ по лемме 6 получаем:

$$
H\left(\eta^{\downarrow}\right)=v_{\delta}(S) \odot \eta^{\downarrow}=\delta(E \oplus S) \odot \eta^{\downarrow} \oplus v_{0}(S) \odot \eta^{\downarrow}=\delta(E \oplus S) \odot \eta^{\downarrow} \in \mathcal{A}_{0} .
$$

Получили противоречие с равенством (28). Следовательно, $\left(v_{0}(x), F_{0}(x)\right)=e$.

Теперь покажем, что если $\delta \neq 0$, то $\left(v_{\delta}(x), F_{0}(x)\right)=e\left(\right.$ т.е. $v_{\delta}(S)$ обратима). Так как $\mathcal{A}_{\delta}=\mathcal{A}_{0} \oplus \delta E_{1}^{\downarrow}$ и $\operatorname{dim} \mathcal{A}_{0}=m-1$, то $\mathcal{A}_{\delta}$ содержит линейно независимую систему из $m$ векторов. Пусть $w_{1}^{\downarrow}, \ldots, w_{m}^{\downarrow}-$ такая система для множества $A_{\delta\left(e \oplus F_{1}(e)\right)}$. Следовательно, из равенства $v_{\delta}(S) \odot \mathcal{A}_{\delta}=\mathcal{A}_{\delta\left(e \oplus F_{1}(e)\right)}$ получаем, что существует решение $X \in P_{m, m}$ уравнения $v_{\delta}(S) \odot X=\left(w_{1}^{\downarrow}, \ldots, w_{m}^{\downarrow}\right)$, т. е. $v_{\delta}(S)$ обратима.

Наконец, заметим, что условие $F_{1}(e) \neq e$ следует из равенства $\left(v_{0}(x), F_{0}(x)\right)=e$. Теорема 1 доказана.

\section{Заключение}

Преимуществом рекурсивных методов построения преобразований является простота реализации и компактность задания. Для реализации 
подстановки $(5)$ на пространстве $P^{(m)}$ достаточно хранить в памяти лишь набор коэффициентов многочлена $F(x)$. Трудоёмкость вычисления значения подстановки $\pi_{F}$ равна трудоёмкости вычисления $m$ подряд идущих знаков линейной рекуррентной последовательности с характеристическим многочленом $F(x)$.

Полученные в работе результаты показывают, что, используя только линейный рекуррентный закон и разрядные функции кольца Галуа, можно строить классы нелинейных подстановок на пространстве сколь угодно большой размерности.

Автор выражает глубокую признательность профессору А.А. Нечаеву за постановку задачи и внимание к проводимым исследованиям.

\section{Список литературы}

[1] Аборнев А. В., "Построение подстановок с использованием разрядно-подстановочных преобразований модуля над кольцом Галуа характеристики 4", Прикл. дискр. матем., 1 (2014), 9-19.

[2] Кузьмин А. С., Нечаев А. А., “Линейные рекуррентные последовательности над кольцами Галуа", Алгебра и логика, 34:2 (1995), 169-189.

[3] Nechaev A. A., Abornev A. V., "Nonlinear permutations on a space over a finite field induced by linear transformations of a module over a Galois ring", Математические вопросы криптографии, 4:2 (2013), 81-100.

[4] Никонов В. Г., Саранцев А. В., “Методы компактной реализации биективных отображений, заданных регулярными системами однотипных булевых функций", Вестник РУДН, Сер. Прикл. и компъют. матем., 2:1 (2003), 84-95. 